DR. GIACOMO BEGNONI (Orcid ID : 0000-0003-1292-3745)

DR. FEDERICA MUSTO (Orcid ID : 0000-0002-9803-078X)

Article type : Original Article

\title{
Electromyographic analysis of the oral phase of swallowing in subjects with and without atypical swallowing: a case control study
}

\author{
Swallowing's oral phase activation pattern
}

Begnoni Giacomo¹, giacomo.begnoni@unimi.it

Cadenas de Llano-Pérula Maria², maria.cadenas@uzleuven.be

Willems Guy², guy.willems@uzleuven.be

Pellegrini Gaia ${ }^{1}$, gaiapellegrini.perio@gmail.com

Musto Federica ${ }^{1}$, federica.musto@hotmail.com

Dellavia Claudia ${ }^{1}$, claudia.dellavia@unimi.it

${ }^{1}$ Department of Biomedical, Surgical and Dental Sciences, University of Milan, Via Luigi Mangiagalli 31, 20133 Milan, Italy.

${ }^{2}$ Department of Oral Health Sciences - Orthodontics, KU Leuven and Dentistry, University Hospital Leuven, Kapucijnenvoer 7, 3000 Leuven, Belgium.

\section{*Corresponding author:}

Dr Giacomo Begnoni, DDS, PhD

Department of Biomedical, Surgical and Dental Sciences

University of Milan

Via Luigi Mangiagalli 31

20133

Milano

giacomo.begnoni@unimi.it

Tel: +390250315404

This article has been accepted for publication and undergone full peer review but has not been through the copyediting, typesetting, pagination and proofreading process, which may lead to differences between this version and the Version of Record. Please cite this article as doi: $10.1111 /$ joor.12826

This article is protected by copyright. All rights reserved. 


\section{Abstract}

\section{Background:}

Swallowing is a complex physiologic function developing mostly in the first years of life. After 6 years old, if mature deglutition is not achieved, swallowing persists as "atypical swallowing" (AS).

\section{Objective:}

The aim of this study was to detect any electromyographical differences in the muscular activation pattern in patients with and without AS.

\section{Materials and Methods:}

38 adolescents and young adults were selected for this study: 20 with atypical swallowing (AS group) and 18 without ( $C$ group). Standardized surface electromyographic analysis was performed by the same operator to detect the activity of masseter (MM), temporalis (TA) and submental (SM) muscles. A Student-t test for unpaired data was carried out to detect differences between the AS and $C$ groups. A 1-way ANOVA was performed to detect any differences between the three couples of muscles.

\section{Results:}

When compared to controls, AS patients showed a significantly longer duration of activity for each couple of muscles and for the whole duration of swallowing act $(p<.0001)$ as well as lower intensity of the SM activity $(p<.05)$ than controls. Within the AS and C groups, masticatory muscles (MM and TA) showed lower duration of activation $(p<.01)$ and lower intensity of the spike $(p<.0001)$ than SM. Within the $\mathrm{C}$ group, masticatory muscles also reached their activation spike earlier (1-way ANOVA, $p<.01)$ than SM.

\section{Conclusion}

Two different muscular performance models have been defined: patients with AS showed a longer activity of all the muscles involved with a lower intensity of SM activity than that of controls.

Keywords: atypical swallowing, surface electromyography, sEMG, deglutition, orthodontics

\section{Introduction}

Swallowing has been defined as a complex physiological act that occurs thousands of times per day and is fundamental for the development of the stomatognathic system ${ }^{1,2}$. Hypothesis about the effects of tongue's variations in size $e^{3-5}$, function ${ }^{6-8}$ and posture ${ }^{1,9,10}$ on the oral development and function and teeth position have been provided previously.

In the first years of life, swallowing is characterized by a lack of activation of the masticatory muscles and by a major activity of the orbicularis muscle with interposition of the tongue between the alveolar ridges of upper and lower incisors. In this way a negative intraoral pressure is provided, and the suction effect and the deglutition are allowed ${ }^{11}$. In continuity with the last fetal period, this kind of swallowing is mainly reflex and characterized by peristaltic muscular contraction waves, similar to

This article is protected by copyright. All rights reserved. 
the ones observed in the gut ${ }^{12}$. For this reason, authors talk about "visceral swallowing", also known as "infantile swallowing". ${ }^{13,14}$

From six months to six years of age the visceral swallowing pattern changes gradually. At 6 months, a primitive swallowing-breathing coordination appears ${ }^{15}$. From that moment and up to 24 months of age, when the infant starts to ingest solid and semi-solid foods, the oral phase of swallowing develops and most of the oral reflexes stop ${ }^{16}$. Finally, between 2 and 6 years old, the mastication function is improved ${ }^{17}$, and the characteristic tongue thrust of the infantile swallowing is slowly stopped $^{1,18}$. The maturation of a more conscious and voluntary swallowing action is responsible for the change from infantile to adult or somatic swallowing. If this maturation is not achieved, infantile swallowing persists as "atypical swallowing"(AS) or tongue thrust, which is considered dysfunctional after the sixth year of life ${ }^{1}$.

The etiology of AS seems to be multifactorial, involving environmental, hereditary factors, oral and allergic diseases ${ }^{11,13,19,20}$. It has an incidence of 50 percent at 5 years old, decreasing to $38 \%$ in early mixed dentition, dropping to $30 \%$ once the second dentition is completed and persisting in about $15 \%$ of adults. This suggests that functional traits change during growth and benefit from the best occlusal stability achieved at the end of the second dentition ${ }^{1,19,21}$.

In clinical practice, the diagnosis of normal or atypical swallowing is normally performed by the observation during swallowing of the presence of a tongue thrust associated with facial myofunctional alterations. ${ }^{22,23}$ However, the analysis of the muscular activity involved in swallowing could help the clinician in the diagnosis, prognosis, and interception of AS, as well as in evaluating the effects of treatment, either myofunctional or orthodontic. Surface electromyography (sEMG) represents an easy method for detecting the muscle activity involved during swallowing ${ }^{24-27}$. During the oral phase of swallowing, masticatory (masseter ( $M M)$ ), temporalis (TA) and pterygoid muscles) and opening muscles (submental muscles $(\mathrm{SM})$ ) stabilize the mandible during oral closure and tongue elevation ${ }^{24,28}$. SEMG analysis in AS patients of the activity of these muscles has already been proposed by previous studies, but the sEMG protocols were not standardized. ${ }^{29,30}$.

Although some authors have suggested that the electromyographical signal has technical and biological limits that could make a comparative and longitudinal analysis difficult ${ }^{31-33}$, the standardized SEMG (sSEMG) procedure excludes these features and permits data evaluation ${ }^{31,34}$. Recently, a standardized protocol on muscular activity of MM, TA and SM during saliva swallowing has been defined in healthy adult subjects ${ }^{32}$, with preliminary normalized data being available ${ }^{35}$. According to this proposed muscular model, during swallowing there is a first activation of the masticatory muscles ( $\mathrm{MM}$ and TA) with moderate intensity followed by a longer activation of SM muscles with a higher intensity ${ }^{35}$.

The aim of this study is to assess if differences in the muscular activity pattern during swallowing occurs between patients with atypical or normal swallowing using the standardized surface electromyography protocol.

This article is protected by copyright. All rights reserved. 


\section{Materials and Methods}

The present clinical work is an observational case-control study of two groups presenting a different swallowing pattern (atypical swallowing - AS and normal swallowing-C), that were analyzed with standardized electromyography.

\section{Patient selection}

Recruitment of the atypical swallowing group (AS group)

25 adolescents and young adults coming to the Department of Biomedical, Surgical and Dental Sciences of the Università degli Studi di Milano were enrolled in this study as experimental group (AS). The diagnosis of normal or atypical swallowing was performed by the same operator by observing the patients during swallowing. This operator was a specialized orthodontist familiar with the clinical evaluation of oral dysfunctions. Hyper activation of lips or of orbicularis muscles, facial muscle tension and an abnormal movement of head and mandible inclined the operator towards the diagnosis of atypical swallowing. If the presence of tongue thrust was observed without any doubts and was then associated with the myofunctional alterations already listed, the diagnosis was considered definitive. Tongue thrust was defined as protrusion of the tongue between upper and lower incisors or cuspids during swallowing ${ }^{37}$.

Inclusion criteria for this group were: second dentition complete with at least 28 teeth present, no temporo-mandibular disorders (TMD), no oral breathing, no oral bad habits, overjet and overbite between 0 and $5 \mathrm{~mm}$, no vertical or sagittal skeletal discrepancies, dental crowding inferior to $6 \mathrm{~mm}$ and AS (Figure 1).

Recruitment of the control group (C group)

18 adolescents and young adults coming to the Department of Oral Health Sciences - Orthodontics of the University Hospitals Leuven, Belgium were recruited for the control group (C). The diagnosis of normal swallowing was performed by the same operator as for the AS group. If the patients during swallowing did not show any alteration in the activation of lips, orbicularis and facial muscles and movement of the mandible, and no tongue thrust was observed, then their swallowing was considered as normal.

All patients showed the following characteristics: first molar class, second dentition complete with at least 28 teeth present, no temporo-mandibular disorders (TMD), absence of oral habits or dysfunctions, overjet and overbite between 1 and $4 \mathrm{~mm}$, no vertical or sagittal discrepancies.

Exclusion criteria for both AS and C groups were: no periodontal disease, no lateral crossbite, no active medication intake, no active orthodontic therapy, no occlusal overlay, no fixed prosthetic, no active caries, no endodontic treatment and no conservative or oral surgical therapy within the last three months.

This article is protected by copyright. All rights reserved. 
All patients enrolled in this study were invited to sign an informed consent and the study protocol was approved by the local ethic committees of each University. The study of AS group was registered with the national code DG-EMG-2016 and the study of the C group was registered with the national code B322201316750.

\section{Electromyographic analysis}

Surface EMG activity was recorded in order to analyze the activation pattern of the MM, TA and SM muscles, using a standardized method with a computerized instrument (Easymyo; 3 Technology S.r.l., Udine, Italy), whose electrode type and procedure of analysis have been described previously ${ }^{35}$.

The bipolar surface electrodes $(21 \times 41 \mathrm{~mm}, 20 \mathrm{~mm}$ inter- electrode distance; F3010; Fiab, Firenze, Italy) were positioned on the muscular bellies parallel to the muscular fibers for the bilateral recording as follows ${ }^{35}$ :

1. MM. The electrodes were fixed parallel to the exocanthion-gonion line and with the upper pole of the electrode under the tragus-labial commissural line.

2. TA. The electrodes were fixed vertically along the anterior margin of the muscle (corresponding to the fronto-parietal suture).

3. SM. The electrodes were placed in the submental area, paramedian to the midline and lightly diverging, $1 \mathrm{~cm}$ posterior to the mental symphysis ${ }^{32}$.

A disposable reference electrode was applied to the forehead or earlobe of the patient. To reduce skin impedance, the skin was carefully cleaned with alcohol prior to electrode placement, allowing the conductive paste to adequately moisten the skin.

\section{ssEMG Measurements}

The ssEMG analysis was composed of three parts (repeated twice):

1. Masticatory muscles standardization procedures: Two 10-mm-thick cotton rolls were positioned on the mandibular second premolars/first molars of each participant, and a 5second maximum voluntary contraction (MVC) was recorded to standardize TA and MM sEMG signal. The mean ssEMG potential obtained in the first acquisition was set at $100 \%$, and all further ssEMG potentials were expressed as a percentage of this value $(\mu \mathrm{V} / \mu \mathrm{V} \times$ 100).

2. Submental muscles standardization procedures: participants were asked to push their tongue against the palate (without teeth clenching), and a 5-second SSEMG SM activity was recorded. All further ssEMG potentials were expressed as a percentage of this value $(\mu \mathrm{V} / \mu \mathrm{V}$ $\times 100)$.

3. Saliva swallowing: after drinking $20 \mathrm{cc}$ of water, participants were asked to wait 30 seconds, bring their teeth in contact during swallowing the saliva spontaneously accumulated and keep them in rest position (with no occlusal contacts). At the end, a 5-second ssEMG activity was recorded.

This article is protected by copyright. All rights reserved. 
A 90 seconds break period elapsed between the 2 acquisitions. For each acquisition, (that last 5seconds each), the dedicated software automatically selected 3 seconds as the most efficient recursive period length over which to calculate the simple moving average in order to stabilize the signal. ${ }^{38}$

During the tests, participants were asked to perform to the best of their ability, to avoid head and neck movements and to maintain a relaxed facial expression to reduce cross-talks. During the recordings, participants sat in a comfortable office-type chair with a straight posture, feet flat on the floor and arms resting on their legs. All electromyographical acquisitions were made by the same operator.

\section{ssEMG Data Analysis}

The ssEMG waves (Figure 2) were amplified, digitalized, digitally filtered and interfaced with a computer which presented the data graphically and recorded them on magnetic media for further quantitative and qualitative analyses:

1. POC (\%, percentage overlapping coefficient): this index represents the symmetrical activation of each couple of muscles ranging between $0 \%$ and $100 \%$. When 2 paired muscles (MM, TA, SM) contract with perfect symmetry, a POC of $100 \%$ is obtained. Details on the calculation of the POC index have been described in our previous study ${ }^{31}$.

2. IMPACT (\%, standardized activity index): activity index that quantifies the total muscular recruitment (MM, TA, SM) during swallowing relative to the standardization procedures (see above), computing the mean total muscle activities as the integrated areas of the sEMG potentials over time. Details on the calculation of the IMPACT index have been described in our previous study ${ }^{39}$.

From the swallowing wave of each muscle the following extra measurements were taken:

3. Duration of activation of each couple of muscles (MM, TA, SM): considered as the interval in which muscle activity was higher than $10 \%$ of its standardization procedure. Values below this threshold were considered muscular basal activity as reported previously ${ }^{35}$.

4. Duration of the whole swallowing test: described as the interval between the beginning of the first muscle (MM, TA or SM) activation and the end of the last muscle activation (MM, TA or SM).

5. Position of the spike of each couple of muscles relatively to its total duration of activity: reported as a percentage of the duration of the activation of each couple of muscles; when a continuous wave is present, and the spike position is unclear, it is considered as the middle point between the start and the end of the electromyographical wave. The term "spike" indicates the electromyographical wave with the highest amplitude in the period observed.

6. Intensity of the spike of each couple of muscles: the percentage of activity of MM, TA, SM relative to the standardization procedures expressed in correspondence of the spike point during swallowing.

This article is protected by copyright. All rights reserved. 
One operator established the diagnosis and another one made the SEMG analysis. Then, all electromyographic data were processed blindly with respect to the patient belonging to one or the other group.

\section{Statistical Analysis}

To assess the reliability of the measurements recorded, a Student t-test for paired data was performed and no significant results were detected ( $p>05$ ).

Descriptive statistics were computed for all SSEMG indexes for swallowing. To compare data relative to the electromyographic indexes between AS and C, a Student $t$-test for unpaired data was performed. In addition, to detect any significant differences within the AS and $C$ group mean values were compared by 1-way ANOVA analyses of variance, followed by post hoc tests. ${ }^{39}$

The null hypothesis was that no differences in duration and intensity of activation of the different couples of muscles would be detectable. For all statistical tests, significance was set at $5 \%(p<0.05)$, with a beta error (Type II) larger than $0.95^{34}$.

\section{Results}

A final group of 20 AS patients (4 males and 16 females, mean age $17.85 \pm 4.80$ ) did comply with the inclusion criteria and was included in the study. For the $\mathrm{C}$ group a total of 18 patients ( 8 males and 10 females, mean age $17.28 \pm 2.56$ ) were enrolled. No significant age differences were detected between groups. Data on age, overjet (OVJ) and overbite (OVB) are summarized in Table 1.

According to the Student $t$-test, AS patients showed a significantly $(p<.01)$ higher overjet (mean 3.78 $\pm 2.28)$ and a significantly $(p<.05)$ lower overbite $(0.98 \pm 1.57)$ compared to the $C$ group $(2.14 \pm 0.33$; $1.81 \pm 0.60$ ). Values for POC, IMPACT, duration of activation of each couple of muscles, duration of the whole exercise, position of the spike and intensity of the spike in the AS and C groups are reported in Table 2.

No statistical differences were recorded between the AS and C groups with the Student-t-test for both spike position and intensity. When analyzing both groups separately, statistical differences were recorded with the 1-way ANOVA and between the different couples of muscles for intensity of the spike in both groups and for spike position in the $C$ group.

At the post hoc tests masticatory muscles (MM and TA) showed an earlier $(p<.01)$ and lower $(p<.0001)$ spike intensity compared to SM (Table 2; Figure 3 ).

\section{Discussion}

ssEMG is considered a helpful tool in the analysis of masticatory muscle function and its use in measuring the activity of MM, TA and SM during clenching has been widely studied. ${ }^{31,32,34,38,40}$ In contrast, only a few studies have been performed regarding muscular behavior during swallowing by means of ssEMG ${ }^{32,35}$ and the recruitment of masticatory and submental muscles during this function is still unclear.

This article is protected by copyright. All rights reserved. 
The aim of this work was to compare the muscular activation pattern during swallowing in dysfunctional and normal patients and to detect if significant differences emerged. To do so, we focused on the muscular activity during swallowing by asking the patient to keep the teeth in contact while swallowing saliva as it can be considered as the most common physiological activity and it has been demonstrated a reliable test in healthy subjects ${ }^{32,35}$.

The most relevant outcome of the present work is related to the duration of muscular activation during swallowing. The duration of the whole swallowing act is significantly $(p<.0001)$ longer in AS $(2.23 \mathrm{sec})$ than in $\mathrm{C}(1.30 \mathrm{sec})$ patients, confirming the values obtained in previous studies ${ }^{29,41-43}$. The duration of the activity of the couples of muscles (TA, MM, SM) was also significantly longer in AS than in $C$ patients, but in both groups a similar activation pattern was found with an earlier and lower activation of the masticatory muscles than that of the SM. Furthermore, a significant difference between the couples of muscles was detected within the groups. MM showed the shortest, SM the longest and TA an intermediate duration confirming the data obtained in our previous work ${ }^{35}$, which already suggested that SM works from the earliest stage until the pharyngeal phase of swallowing ${ }^{35}$. Interestingly, the duration of activation in the control group seems to be shorter than that obtained in this previous work, where a group of healthy adult patients not previously treated with orthodontic devices was analyzed ${ }^{35}$. This may be explained by the differences in age of the study population, since the present control group includes younger subjects than the previous one. This suggests that the age may influence the duration of activation of the different couples of muscles.

The POC index expresses the percentage of activity of each couple of muscles by comparing the normal function recorded with the SEMG with the function performed by the same muscles during the standardization procedure. Normally, a good symmetrical activation for each couple of muscles is considered when the POC index is about $80-85 \% .{ }^{31}$ In this study, POC indexes showed a lower level of symmetry in activation compared to those recorded during clenching ${ }^{32}$ for all the investigated muscles. This turned out to be even lower in AS compared to the C group, even if not significantly. In accordance with previous studies ${ }^{35,44}$ these data confirm that the swallowing act is an unbalanced activity which makes good muscle symmetry difficult to achieve ${ }^{44}$ especially considering the complex system of neural central control located in the brain stem (central pattern generator nuclei and the high number of muscles involved ${ }^{28}$. POC data for of the control group were similar to that of our previous study ${ }^{35}$.

IMPACT values for masticatory muscles were similar between groups while the value for SM resulted significantly higher $(p<.05)$ in the $C$ group than in the AS group. In control subjects, the activity provided by the masticatory muscles was similar to that performed in the AS group, whereas SM seem to be more involved during swallowing in C than in AS patients, a finding also observed by Vaiman et $\mathrm{al}^{27}$. Differently, a lower significant activity is provided by SM in AS than in C patients. During swallowing, SM are activated with tongue movement against the palate to stiffen the mouth floor $^{32}$. It should also be added that the activity of all measured muscles was always lower than $30 \%$ of their maximal activity. Similar MM, TA and SM activity was detected within the AS group, which was initially surprising as activity for the masticatory muscles has been found to be $30-40 \%$ lower than that of SM by other studies ${ }^{27,30}$. The difference may lie in the standardized procedure followed in our research. Before swallowing, patients were asked to keep the teeth in contact to ensure the

This article is protected by copyright. All rights reserved. 
reproducibility of the method ${ }^{35}$. This guided swallowing may have encouraged AS patients to make a greater effort to maintain the teeth in contact than what they would during spontaneous swallowing, opening the possibility of a consequently higher activity of the masticatory muscles than expected. ${ }^{27,30}$.

A great dispersion around the mean suggests that a clear, well-defined activation pattern is difficult to be identified and by increasing the sample size it will be possible to further define the pattern of muscle recruitment during swallowing. Nevertheless, mean values are similar in both groups and even comparable to those obtained in our previous work, which indicates that ssEMG is a helpful tool in defining a common trend of muscular activation. Furthermore, considering the complexity of the characteristics involved in patients that show myofunctional disorders such as atypical swallowing $^{22}$, this standardized procedure can be used to get a more accurate diagnosis of AS, as well as to evaluate the effect of treatment on the muscular pattern during swallowing.

Regarding spike position, there is a common activation trend characterized by a first activation of $\mathrm{MM}$, an intermediate peak of TA, and a last peak of SM (around $38 \%, 45 \%$ and $50-55 \%$ of the whole activity respectively). A significant difference on spike position $(p<.01)$ is observed between masticatory and SM muscles in healthy subjects, whereas no difference is observed in AS patients. This suggests that spike positions in the AS group are almost overlapped and therefore the activation pattern is less defined. In our previous study ${ }^{35}$, the spike position of MM, was similar to the one found in this study (36\%) while the positions of TA and SM occurred earlier (36\% and $42 \%$ respectively) than the ones observed in the present investigation. In our previous work the intensity of the spike was also similar ${ }^{35}$ to the present one: MM showed the lowest value, SM the highest and TA recorded intermediate values (around $50 \%, 72 \%$ and $60 \%$ respectively). Nevertheless, significant lower $(p<.001)$ spike intensity was found in masticatory (MM, TA) than SM both groups.

The different activation patterns of masticatory (MM and TA) and submental muscles is confirmed in Figure 3, which summarizes the activity of the three couples of muscles in each group (AS and C). For ease of reading, SM starts at the origin of the coordinate because this was the first couple of muscles to be activated in almost all the subjects. The masticatory muscles were activated later in both groups. In the AS group both masticatory couples of muscles (MM and TA) were activated $0.3 \mathrm{sec}$ after the SM, whereas in C group TA and MM muscles were activated respectively 0.15 and 0.2 seconds after the SM.

Swallowing is a variable action between subjects, therefore EMG values have been reported to show a high inter individual variability ${ }^{24,44}$, which is confirmed also in this study, especially for the Impact index and intensity of the spike of MM and TA. This behavior could be linked to the voluntary action (under cortical control) of jaw elevator muscles, which are recruited for the stabilization of the mandible. Future analysis on larger samples will be necessary to confirm these preliminary data.

\section{Conclusion}

ssEMG is a valid, non-invasive, rapid and reliable method to detect muscular behavior of masticatory muscles involved in the first phase of swallowing, also in patients with atypical swallowing.

This article is protected by copyright. All rights reserved. 
The main findings of this study are:

1. The whole swallowing act has a longer duration in patients with AS than in control subjects;

2. The duration of activity for all the couples of muscles is longer for AS than for control subjects;

3. Spike positions of TA, MM and SM are overlapped in the AS group, being well defined in the C group;

4. There is a significantly lower activity index (IMPACT) of SM in AS patients than in control subjects.

5. A different muscular activity pattern seems to be observed between two groups of subjects.

\section{Disclosure}

The authors certify that there is no financial support for this research and the authors have stated explicitly that there are no conflicts of interest in connection with this article. The study protocol was approved by the DG-EMG-2016 and B322201316750.

\section{References}

1. Proffit WR, Fields HW, Sarver DM. Contemporary Orthodontics. Elsevier/Mosby; 2013.

2. Begnoni G, Serrao G, Musto F, Pellegrini G, Triulzi FM, Dellavia C. Craniofacial structures' development in prenatal period: An MRI study. Orthod Craniofac Res. 2018;21(2):96-103.

3. Hotokezaka H, Matsuo T, Nakagawa M, Mizuno A, Kobayashi K. Severe dental open bite malocclusion with tongue reduction after orthodontic treatment. Angle Orthod. 2001;71(3):228-236.

4. Liu Z-J, Shcherbatyy V, Gu G, Perkins JA. Effects of tongue volume reduction on craniofacial growth: A longitudinal study on orofacial skeletons and dental arches. Arch Oral Biol. 2008;53(10):991-1001.

5. Turner S, Nattrass C, Sandy JR. The role of soft tissues in the aetiology of malocclusion. Dent Update. 1997;24(5):209-214.

6. Alexander S, Sudha P. Genioglossis muscle electrical activity and associated arch dimensional changes in simple tongue thrust swallow pattern. J Clin Pediatr Dent. 1997;21(3):213-222.

7. Fuhrmann RA, Diedrich PR. B-mode ultrasound scanning of the tongue during swallowing. Dentomaxillofac Radiol. 1994;23(4):211-215.

8. Lowe AA. Correlations between orofacial muscle activity and craniofacial morphology in a sample of control and anterior open-bite subjects. Am J Orthod. 1980;78(1):89-98.

9. Karacay S, Akin E, Ortakoglu K, Bengi AO. Dynamic MRI evaluation of tongue posture and deglutitive movements in a surgically corrected open bite. Angle Orthod. 2006;76(6):1057-1065.

10. Proffit WR. Equilibrium theory revisited: factors influencing position of the teeth. Angle Orthod. 1978;48(3):175-186.

11. Schindler O, Ruoppolo G, Schindler A, Omega. Deglutologia. Omega; 2011.

This article is protected by copyright. All rights reserved. 
12. Gewolb IH, Vice FL. Maturational changes in the rhythms, patterning, and coordination of respiration and swallow during feeding in preterm and term infants. Dev Med Child Neurol. 2006;48(7):589-594.

13. Van Dyck C, Dekeyser A, Vantricht E, et al. The effect of orofacial myofunctional treatment in children with anterior open bite and tongue dysfunction: a pilot study. Eur J Orthod. 2016;38(3):227-234.

14. Peng C-L, Jost-Brinkmann P-G, Yoshida N, Miethke R-R, Lin C-T. Differential diagnosis between infantile and mature swallowing with ultrasonography. Eur J Orthod. 2003;25(5):451-456.

15. Hall KD. Pediatric Dysphagia Resource Guide. Singular/Thomson Learning; 2001.

16. Gisel EG. Effect of food texture on the development of chewing of children between six months and two years of age. Dev Med Child Neurol. 1991;33(1):69-79.

17. Papargyriou G, Kjellberg $\mathrm{H}$, Kiliaridis $\mathrm{S}$. Changes in masticatory mandibular movements in growing individuals: a six-year follow-up. Acta Odontol Scand. 2000;58(3):129-134.

18. Schwaab LM, Niman CW, Gisel EG. Tongue movements in normal 2-, 3-, and 4-year-old children: a continuation study. Am J Occup Ther. 1986;40(3):180-185.

19. Proffit WR. On the aetiology of malocclusion. The Northcroft lecture, 1985 presented to the British Society for the Study of Orthodontics, Oxford, April 18, 1985. Br J Orthod. 1986;13(1):1-11.

20. Saccomanno S, Antonini G, D'Alatri L, D'Angeloantonio M, Fiorita A, Deli R. Case report of patients treated with an orthodontic and myofunctional protocol. Eur J Paediatr Dent. 2014;15(2 Suppl):184186.

21. Ovsenik M, Farcnik FM, Korpar M, Verdenik I. Follow-up study of functional and morphological malocclusion trait changes from 3 to 12 years of age. Eur J Orthod. 2007;29(5):523-529.

22. de Felicio CM, Folha GA, Ferreira CLP, Medeiros APM. Expanded protocol of orofacial myofunctional evaluation with scores: Validity and reliability. Int J Pediatr Otorhinolaryngol. 2010;74(11):1230-1239.

23. de Felicio CM, Medeiros APM, de Oliveira Melchior M. Validity of the 'protocol of oro-facial myofunctional evaluation with scores' for young and adult subjects. J Oral Rehabil. 2012;39(10):744753.

24. Vaiman M, Eviatar E, Segal S. Surface electromyographic studies of swallowing in normal subjects: a review of 440 adults. Report 3. Qualitative data. Otolaryngol Head Neck Surg. 2004;131(6):977-985.

25. Vaiman M, Eviatar E, Segal S. Surface electromyographic studies of swallowing in normal subjects: a review of 440 adults. Report 2. Quantitative data: amplitude measures. Otolaryngol Head Neck Surg. 2004;131(5):773-780.

26. Vaiman M, Eviatar E, Segal S. Surface electromyographic studies of swallowing in normal subjects: a review of 440 adults. Report 1. Quantitative data: timing measures. Otolaryngol Head Neck Surg. 2004;131(4):548-555.

27. Vaiman M. Standardization of surface electromyography utilized to evaluate patients with dysphagia. Head Face Med. 2007;3:26.

28. Ertekin C, Aydogdu I. Neurophysiology of swallowing. Clin Neurophysiol. 2003;114(12):2226-2244.

29. Ciavarella D, Mastrovincenzo M, Sabatucci A, Parziale V, Chimenti C. Effect of the Enveloppe Linguale

This article is protected by copyright. All rights reserved. 
Nocturne on atypical swallowing: surface electromyography and computerised postural test evaluation. Eur J Paediatr Dent. 2010;11(3):141-145.

30. Stormer K, Pancherz H. Electromyography of the perioral and masticatory muscles in orthodontic patients with atypical swallowing. J Orofac Orthop. 1999;60(1):13-23.

31. Ferrario VF, Sforza C, Colombo A, Ciusa V. An electromyographic investigation of masticatory muscles symmetry in normo-occlusion subjects. J Oral Rehabil. 2000;27(1):33-40.

32. Musto F, Rosati R, Sforza C, Toma M, Dellavia C. Standardised surface electromyography allows effective submental muscles assessment. J Electromyogr Kinesiol. 2017;34:1-5.

33. Suvinen $\mathrm{TI}$, Malmberg J, Forster C, Kemppainen P. Postural and dynamic masseter and anterior temporalis muscle EMG repeatability in serial assessments. J Oral Rehabil. 2009;36(11):814-820.

34. Sforza C, Rosati R, De Menezes M, Musto F, Toma M. EMG analysis of trapezius and masticatory muscles: experimental protocol and data reproducibility. J Oral Rehabil. 2011;38(9):648-654.

35. Dellavia C, Rosati R, Musto F, Pellegrini G, Begnoni G, Ferrario VF. Preliminary approach for the surface electromyographical evaluation of the oral phase of swallowing. J Oral Rehabil. 2018;45(7):518-525.

36. McCullough GH, Wertz RT, Rosenbek JC, Mills RH, Ross KB, Ashford JR. Inter- and intrajudge reliability of a clinical examination of swallowing in adults. Dysphagia. 2000;15(2):58-67.

37. Jalaly T, Ahrari F, Amini F. Effect of tongue thrust swallowing on position of anterior teeth. J Dent Res Dent Clin Dent Prospects. 2009;3(3):73-77.

38. Ferrario VF, Tartaglia GM, Galletta A, Grassi GP, Sforza C. The influence of occlusion on jaw and neck muscle activity: a surface EMG study in healthy young adults. J Oral Rehabil. 2006;33(5):341-348.

39. Ferrario VF, Tartaglia GM, Maglione M, Simion M, Sforza C. Neuromuscular coordination of masticatory muscles in subjects with two types of implant-supported prostheses. Clin Oral Implants Res.

2004;15(2):219-225.

40. Dellavia C, Francetti L, Rosati R, Corbella S, Ferrario VF, Sforza C. Electromyographic assessment of jaw muscles in patients with All-on-Four fixed implant-supported prostheses. J Oral Rehabil.

2012;39(12):896-904. doi:10.1111/joor.12002

41. Sonies BC, Parent L, Morrish K, Baum BJ. Durational aspects of the oral-pharyngeal phase of swallow in normal adults. Dysphagia. 1988;3(1):1-10.

42. Ichida T, Takiguchi R, Yamada K. Relationship between the lingual-palatal contact duration associated with swallowing and maxillofacial morphology with the use of electropalatography. Am J Orthod Dentofacial Orthop. 1999;116(2):146-151.

43. Monaco A, Cattaneo R, Spadaro A, Giannoni M. Surface electromyography pattern of human swallowing. BMC Oral Health. 2008;8:6.

44. Pernambuco L de A, Silva $\mathrm{HJ}$ da, Lima LM de, et al. Electrical activity of masseter muscle in young adults during swallowing of liquid. J Soc Bras Fonoaudiol. 2011;23(3):214-219.

This article is protected by copyright. All rights reserved. 
Table 1.

\begin{tabular}{|c|c|c|c|c|}
\hline \multicolumn{2}{|c|}{ Index } & AS group & C group & $\begin{array}{l}\text { Student } t- \\
\text { test }\end{array}$ \\
\hline \multirow[t]{2}{*}{ Age } & Mean & 17.85 & 17.28 & \multirow[t]{2}{*}{ NS } \\
\hline & SD & 4.80 & 2.56 & \\
\hline \multirow[t]{2}{*}{ OVJ } & Mean & 3.78 & 2.14 & \multirow[t]{2}{*}{$\mathrm{p}<.01^{* *}$} \\
\hline & SD & 2.28 & 0.33 & \\
\hline \multirow[t]{2}{*}{ OVB } & Mean & 0.98 & 1.81 & \multirow[t]{2}{*}{$p<.05^{*}$} \\
\hline & SD & 1.57 & 0.60 & \\
\hline
\end{tabular}

Table 1: Mean values and standard deviations (SD) of age, overjet (OVJ) and overbite (OVB) in AS and C groups and the differences between values according to the Student-t-test ( $p<.01 * *$, and $\left.p<.05^{*}\right)$.

This article is protected by copyright. All rights reserved. 
Table 2

\begin{tabular}{|c|c|c|c|c|c|c|c|}
\hline Index & \multicolumn{2}{|c|}{ Muscles Couple } & & 1 way & & 1 way & Student \\
\hline \multirow[t]{6}{*}{ POC (\%) } & TA & Mean & 74.22 & \multirow[t]{6}{*}{ NS } & 78.68 & \multirow[t]{6}{*}{ NS } & \multirow[t]{2}{*}{ NS } \\
\hline & & SD & 8.92 & & 5.15 & & \\
\hline & MM & Mean & 73.69 & & 79.75 & & \multirow[t]{2}{*}{ NS } \\
\hline & & SD & 12.61 & & 4.79 & & \\
\hline & SM & Mean & 79.56 & & 79.71 & & \multirow[t]{2}{*}{ NS } \\
\hline & & SD & 6.97 & & 5.74 & & \\
\hline \multirow[t]{6}{*}{ IMPACT (\%) } & TA & Mean & 23.95 & \multirow[t]{6}{*}{ NS } & 25.09 & \multirow[t]{6}{*}{ NS } & \multirow[t]{2}{*}{ NS } \\
\hline & & SD & 16.30 & & 16.90 & & \\
\hline & MM & Mean & 18.27 & & 17.20 & & \multirow[t]{2}{*}{ NS } \\
\hline & & SD & 10.46 & & 14.41 & & \\
\hline & SM & Mean & 19.90 & & 25.61 & & \multirow[t]{2}{*}{$p<.05^{*}$} \\
\hline & & SD & 6.73 & & 8.98 & & \\
\hline \multirow[t]{6}{*}{ Duration (s) } & TA & Mean & $1.60^{\mathrm{a}}$ & \multirow[t]{6}{*}{$\mathrm{p}<.01 * *$} & $0.96^{\mathrm{a}}$ & \multirow[t]{6}{*}{$\mathrm{p}<.01 * *$} & \multirow{2}{*}{$\begin{array}{l}\mathrm{p}< \\
.01 * *\end{array}$} \\
\hline & & SD & 0.60 & & 0.29 & & \\
\hline & $\mathrm{MM}$ & Mean & $1.48^{\mathrm{a}}$ & & $0.82^{a}$ & & \multirow{2}{*}{$\begin{array}{l}\mathrm{p}< \\
.0001 * * \\
*\end{array}$} \\
\hline & & SD & 0.53 & & 0.23 & & \\
\hline & SM & Mean & $2.07^{b}$ & & $1.12^{b}$ & & $\mathbf{p}<$ \\
\hline & & SD & 0.38 & & 0.21 & & \\
\hline \multirow[t]{2}{*}{ Duration TOT (s) } & & Mean & 2.23 & \multirow[t]{2}{*}{$X$} & 1.30 & \multirow[t]{2}{*}{$x$} & \multirow{2}{*}{$\begin{array}{l}p< \\
.0001 * * \\
*\end{array}$} \\
\hline & & SD & 0.45 & & 0.24 & & \\
\hline \multirow[t]{2}{*}{ Spike position (\%) } & TA & Mean & 44.82 & \multirow[t]{2}{*}{ NS } & $45.17^{a}$ & \multirow[t]{2}{*}{$\mathrm{p}<.01 * *$} & \multirow[t]{2}{*}{ NS } \\
\hline & & SD & 17.94 & & 15.56 & & \\
\hline
\end{tabular}

This article is protected by copyright. All rights reserved. 


\begin{tabular}{|c|c|c|c|c|c|c|c|}
\hline & $\mathrm{MM}$ & Mean & 39.41 & & $38.67^{a}$ & & NS \\
\hline & & SD & 15.40 & & 13.30 & & \\
\hline & SM & Mean & 49.52 & & $57.17^{b}$ & & NS \\
\hline & & SD & 13.1 & & 14.28 & & \\
\hline Intensity of the & TA & Mean & $47.98^{a}$ & $p<$ & $57.11^{a}$ & $\mathbf{p}<$ & NS \\
\hline & & SD & 20.11 & & 12.86 & & \\
\hline & MM & Mean & $50.68^{a}$ & & $50.89^{a}$ & & NS \\
\hline & & SD & 20.01 & & 16.20 & & \\
\hline & SM & Mean & $75.45^{b}$ & & $73.19^{b}$ & & NS \\
\hline & & SD & 10.44 & & 8.40 & & \\
\hline
\end{tabular}

Table 2: Standardized SSEMG indexes during saliva swallowing are shown: POC (\%), impact (\%), duration of activation of each couple of muscles (s) (masseter muscles MM, temporalis muscles TA and submental muscles SM), duration of the whole exercise (TOT) (s), spike position (\%) (calculated as a percentage of the duration of activation of each couple of muscles), intensity of the spike (\%), mean and standard deviation (SD) for MM, TA and SM. Student t-test for unpaired data was performed between AS and $C$ and significance was set at 5\% ( $p<.05)$. A 1-way ANOVA was performed between MM, TA and SM muscles within AS and C groups for all electromyography indexes. Probability of one-way analysis of variance; NS: not significant, $P>0.05$. Means with different superscripts ( $a$ or $b$ ) differ at post hoc tests.

\section{Figures captions}

Figure 1: Frontal view of a patient with AS showing the tip of the tongue between the dental arches.

Figure 2: A- $F$, Example of an electromyographical signal of the muscles activated during saliva swallowing. $X$-axis: time (ms), $Y$ - axis: intensity of activation $(\mu \mathrm{V} / \mu \mathrm{V} \times 100)$. A, right anterior temporalis muscle; $B$, left anterior temporalis muscle; $C$, right masseter muscle; $D$, left masseter muscle; $E$, right submental muscles; $F$, left submental muscles

Figure 3: Graphic representing the position and the intensity of the spike of activation of each couple of muscles during saliva swallowing in group AS (figure 2a) and group C (figure $2 b$ ), considering mean values. MM, TA and SM are reported with different colors. Duration (s) is represented on the abscissae axis and intensity of activity (\%) on the ordinates axis. MM, masseter; SM, submental muscles; TA, anterior temporalis. " $X$ " is located on the $x$ axis at the time of the end of the swallowing act.

This article is protected by copyright. All rights reserved. 

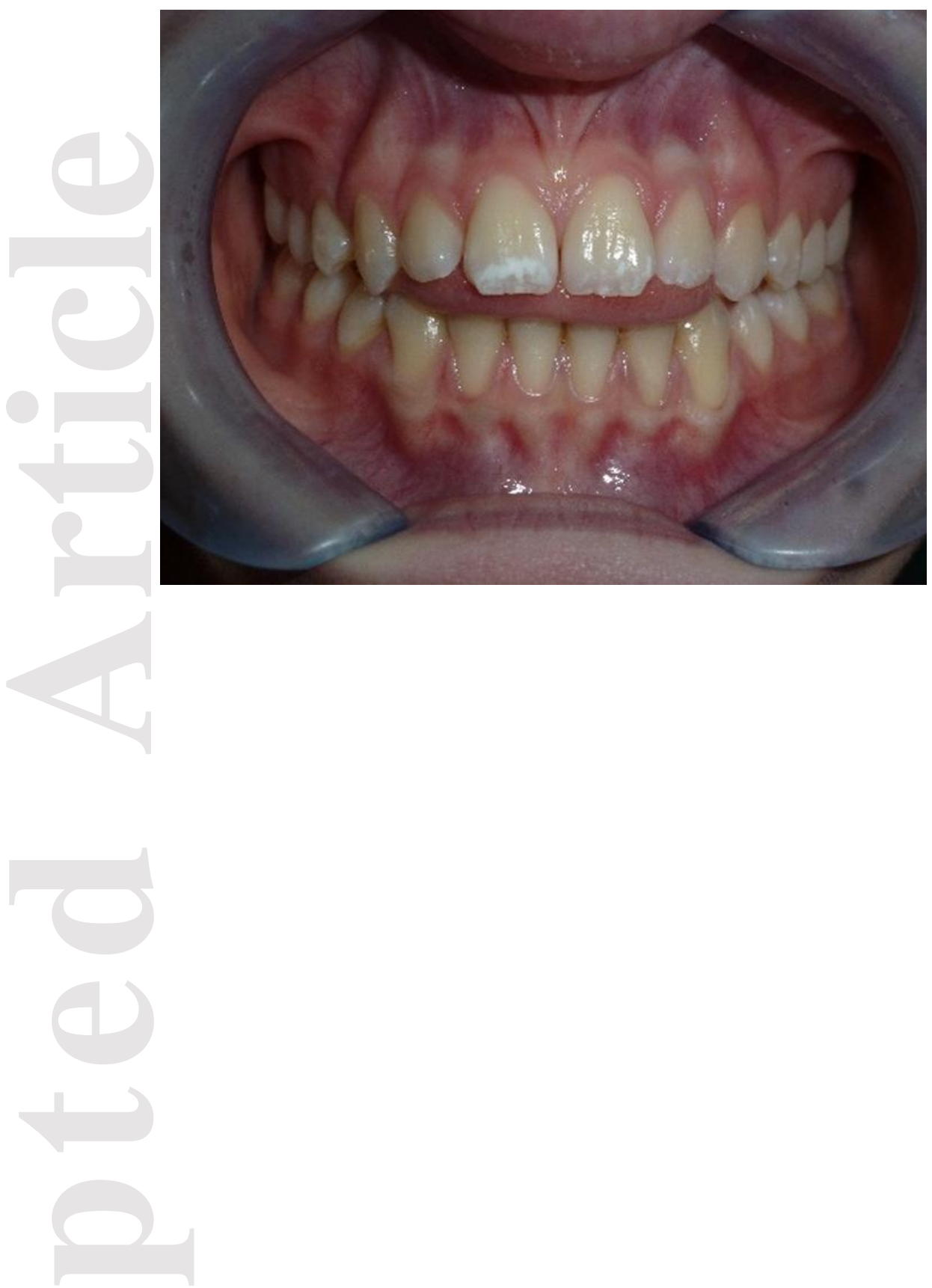

This article is protected by copyright. All rights reserved. 

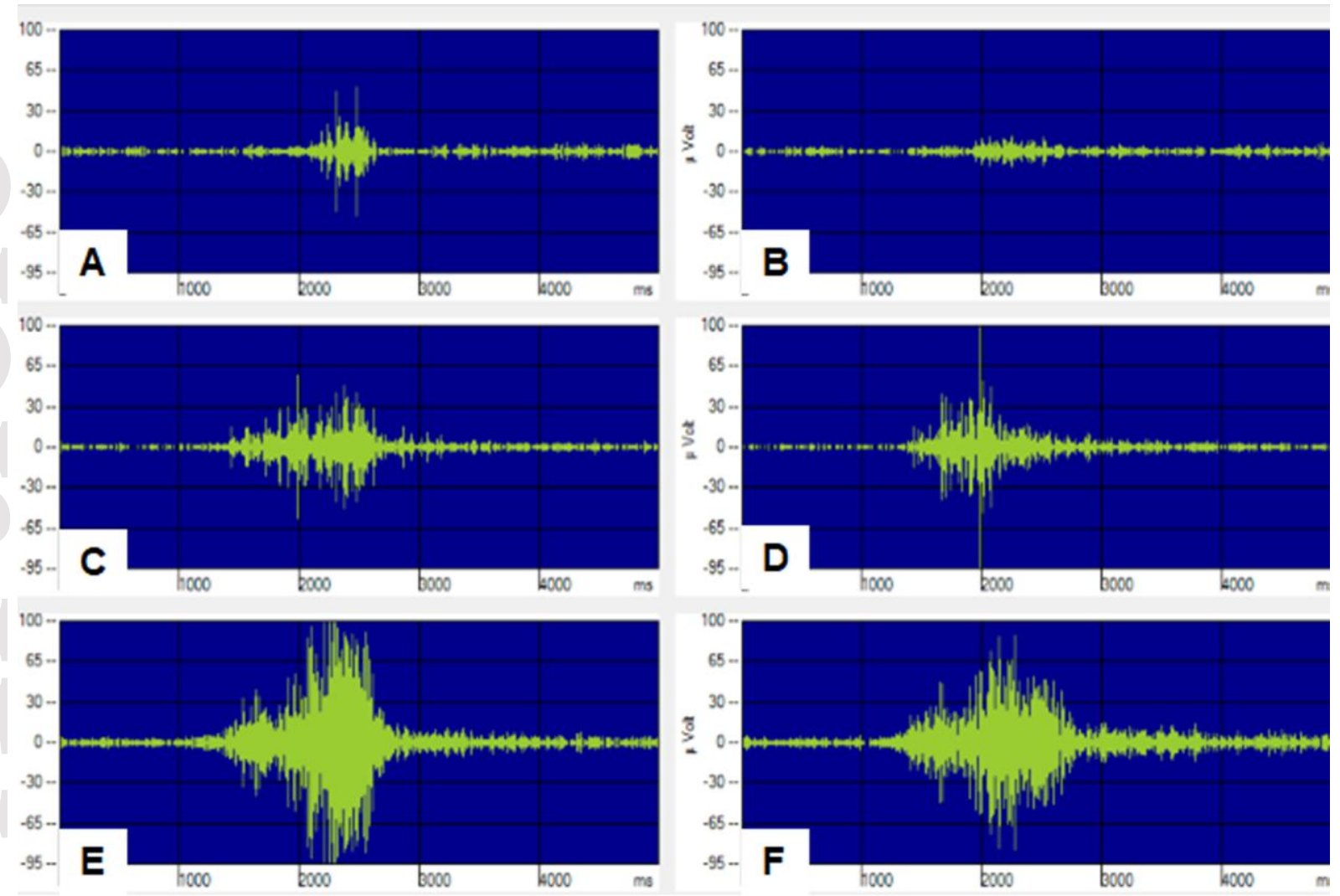

This article is protected by copyright. All rights reserved. 


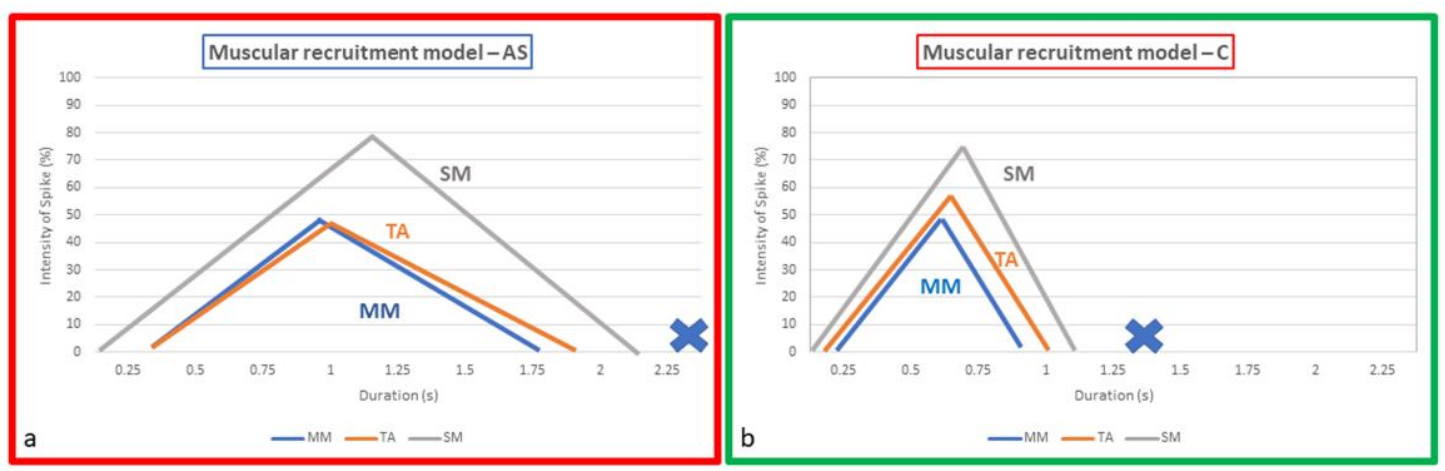

This article is protected by copyright. All rights reserved. 